\title{
Replacement therapy, not recreational tonic
}

\section{Testosterone, widely used as a lifestyle drug, is a medicine and should be kept as such.}

Sir — We read with great interest the News Feature "A dangerous elixir?" (Nature 431, 500-501; 2004) reporting the zeal with which testosterone is being requested by men who apparently view the hormone as a 'natural' alternative to the drug Viagra.

Like Viagra, testosterone replacement therapy — with emphasis on the word 'replacement' - is a solution to a very real clinical problem. There is a distinction between patients whose testosterone has declined to abnormally low levels, and individuals seeking testosterone as a pickme-up. (As clinical and basic researchers interested in the effects of testosterone on the cardiovascular system, we find that many clinicians and scientists also seem unable to make this distinction.) Perhaps it is not surprising that testosterone is turning into a lifestyle drug.

During the Depression, the United States was rife with travelling 'doctors' peddling male tonics and remedies - most harmless, but some as drastic as grafting goat testicles onto humans. Unfortunately, a certain amount of quackery is now perceived to be associated with testosterone therapy, and the increased prescription of the hormone in recent years does little to dispel this notion.

Nonetheless, as your Feature highlights, hypogonadism is a very real clinical condition. It is characterized by abnormally low serum levels of testosterone, in conjunction with symptoms such as mood disturbance, depression, sexual dysfunction, decreased muscle mass and reduced bone-mineral density.

Heightened awareness of hypogonadism, together with the increasing incidence of associated conditions such as obesity, may in part explain the rise in testosterone usage. But the inappropriate prescribing of the hormone is likely to be a contributory factor.

We agree with the experts quoted in your Feature that there is a need for largescale, long-term clinical trials to address several issues associated with testosterone use. For example, testosterone therapy may be beneficial against osteoporosis, heart disease and Alzheimer's disease, but the dangers remain obscure.

Until we know more, both prescribing clinicians and the male population need to be aware that testosterone, like any other medication, should only be administered to patients for whom such therapy is clinically indicated.

Richard D. Jones ${ }^{\star}$ T. Hugh Jones ${ }^{\star}$,

Kevin S. Channer $\dagger$

${ }^{\star}$ Hormone \& Vascular Biology Group, Academic

Unit of Endocrinology, Division of Genomic

Medicine, The University of Sheffield Medical

School, Beech Hill Road, Sheffield S10 2RX, UK

$\dagger$ Faculty of Health \& Well-being,

Sheffield Hallam University,

Collegiate Crescent, Sheffield S10 2BD, UK

\section{DDT still has a role in the} fight against malaria

Sir - Your News story about the Roll Back Malaria campaign ("Struggling to make an impact” Nature 430, 935; 2004) quotes me as claiming that pressure from government and other donors made spraying difficult to push through politically. I am also quoted as saying: "We have had very, very strong lobbying over DDT. We have had to give up." The quotations give the impression that the World Health Organization (WHO) has given up on DDT under the pressure of lobbying. I believe this is misleading.

When interviewed, I explained that we sometimes had to give up trying to convince a specific donor to financially support indoor spraying with DDT, if they flatly refused because of its perceived toxicity and ecological hazard. This has occasionally occurred in countries where the government wished to use DDT, and there was evidence that it was the best option for malaria-vector control.

However, in general terms, the WHO has never given up in its efforts to ensure access to DDT where it is needed. At meetings of the intergovernmental negotiation committee on the Stockholm Convention - which seeks to control the spread of persistent organic pollutants - the WHO has successfully defended the right of countries to use DDT for disease-vector control, if no suitable alternative can be found. The WHO also supports worldwide efforts to develop alternative products and phase in alternative control strategies (www.pops.int/documents/meetings/ inc4/en/inf9/inf9en.pdf).

The Stockholm Convention came into force in May this year. Its exemption allowing restricted and controlled use of DDT according to WHO guidelines is a good example of appropriate international regulation on a difficult dilemma. It is not a compromise but a solution, which ensures that disease-control programmes maintain access to a useful product, while fully respecting the need to prevent environmental damage from persistent organic pollutants, such as DDT.

Allan Schapira

Strategy and Policy Team, Roll Back Malaria

Department, World Health Organization,

Avenue Appia 20, 1211 Geneva 27, Switzerland

\section{Presidential candidates failed peer-review test}

Sir - Peer review has had a bad press recently, but your piece by George W. Bush and John Kerry (Nature 431, 240-243; 2004) shows how valuable it can be.

The article is conspicuously full of unsupported (and unsupportable) claims and deliberately ambiguous statements of the sort that never usually appear in Nature, because no reviewer would allow them. Maybe all statements by politicians should be refereed by scientists.

Of course, this idea also exposes the weakness of peer review. The article would have to be refereed by Bush and Kerry's peers, that is to say disingenuous politicians. Such referees would no doubt insist on removing any shred of clarity and simple truthfulness as contrary to accepted practice.

The principle of peer review is an excellent one, but the practice is only as good as the reviewers.

Robert Insall

School of Biosciences, University of Birmingham, Edgbaston, Birmingham B15 2TT, UK

\section{Benveniste's reputation was not written in water}

Sir - The headline on your News story about the late Jacques Benveniste "'Memory of water' biologist dies after heart surgery" (Nature 431, 729; 2004) reminds readers of the most controversial aspects of his career. His work cannot be fully appreciated by exploring only the second half of his scientific life. We should also remember his active participation in the discovery of platelet-activating factor, a potent pro-inflammatory lipid-derived mediator (J. Benveniste, P. M. Henson and C. G. Cochrane J. Exp. Med. 136, 1356-1377, 1972; and J. Benveniste Nature 249, 581-582, 1974).

Without omitting the more questionable part of his career, he deserves wider attention for this early work. His memory lives on.

Bernard Rothhut

CNRS UMR 6198, UFR Sciences, Université de Reims Champagne-Ardenne, 51687 Reims cedex 2, France 